\title{
Differentiation of Neural Precursors and Dopaminergic Neurons from Human Embryonic Stem Cells
}

\author{
Xiao-Qing Zhang and Su-Chun Zhang ${ }^{*}$ \\ Departments of Anatomy and Neurology, School of Medicine, The Stem Cell Research Program, \\ Waisman Center, and the WiCell Institute, 1500 Highland Avenue, University of Wisconsin-Madison, \\ WI 53705
}

\begin{abstract}
Directed differentiation of human embryonic stem cells (hESCs) to a functional cell type, including neurons, is the foundation for application of hESCs. We describe here a reproducible, chemicallydefined protocol that allows directed differentiation of hESCs to nearly pure neuroectodermal cells and neurons. First, hESC colonies are detached from mouse fibroblast feeder layers and form aggregates to initiate the differentiation procedure. Second, after 4 days of suspension culture, the ESC growth medium is replaced with neural induction medium to guide neuroectodermal specification. Third, the differentiating hESC aggregates are attached onto the culture surface at day 6-7, where columnar neural epithelial cells appear and organize into rosettes. Fourth, the neural rosettes are enriched by detaching rosettes and leaving the peripheral flat cells attached, and expanded as neuroepithelial aggregates in the same medium. Finally, the neuroepithelial aggregates are dissociated and differentiated to nearly pure neurons. This stepwise differentiation protocol results in the generation of primitive neuroepithelia at day $8-10$, neural progenitors at the $2^{\text {nd }}$ and $3^{\text {rd }}$ week, and postmitotic neurons at the $4^{\text {th }}$ week, which mirrors the early phase of neural development in a human embryo. Identification of the primitive neuroepithelial cells permits efficient patterning of region-specific progenitors and neuronal subtypes such as midbrain dopaminergic neurons.
\end{abstract}

\section{Keywords}

Neural induction; Embryonic stem cells; Differentiation; Cell replacement; Dopaminergic neurons

\section{Introduction}

Directed differentiation of specific lineages has been a focal point in the field of human embryonic stem cell (hESC) research $(1,2)$. The differentiation paradigms are key to revealing the cellular and molecular mechanisms underlying early human development, exploring tissue engineering, and devising cell replacement therapy and drug screening. Ideally, the differentiation protocol should be based on the developmental principles and truly directed (controllable). It should also be simple, defined, and reproducible.

We have developed a reproducible, chemically-defined protocol that allows directed differentiation of hESCs to a synchronized population of neuroectodermal cells and then a nearly pure population of neurons $(3,4)$. The design of the protocol rests essentially on the fundamental principle of neuroectodermal induction (5). As such, the specification of neuroepithelial cells and subsequent neuronal and glial differentiation mirrors the time course

*Correspondence: Su-Chun Zhang, MD, PhD Waisman Center, Rm 651 University of Wisconsin 1500 Highland Avenue Madison, WI 53705 Phone: (608) 265-2543 Fax: (608) 263-5267 zhang@waisman.wisc.edu. 
of human embryo development predicted from animal embryological studies and limited human specimens. We avoid any unknown components, such as sera or stroma cells, in the system so that the culture is chemically-defined, allowing flexible modification for mechanistic analyses. Because of the simplicity, the protocol has been proven highly reproducible for the past decade. The neural differentiation is also robust with over $90 \%$ efficiency $(3,4)$. This endows the system particularly amenable for biochemical analysis that often requires materials of large amount. Technically, the adherent colony culture also permits continual observation of morphological transformation along the differentiation processes.

Under this protocol, differentiating cells manifest five morphologically identifiable stages, the ESCs, ESC aggregates, neuroepithelia in the form of neural tube-like rosettes, neural progenitors in neuroepithelial aggregates, and postmitotic neurons. The undifferentiated state of the starting cell population is essential for the successful replication of the protocol. The quality of hESCs is often overlooked when it comes to differentiation. In reality, partially differentiated hESCs will unavoidably result in unsynchronized differentiation, such as the generation of postmitotic neurons within the first week along with neuroepithelial generation. Human ESCs, cultured on mouse embryonic fibroblast (MEF) feeder layers, are usually passaged every 5-6 days before differentiation. At this stage, the ESC colonies are generally uniform in size, and they form aggregates of similar size after detaching from the MEF layers. These ESC aggregates are initially suspended in the ESC growth medium for 4 days to promote cell survival and then the medium is replaced with the serum-free neural induction medium to guide the cells toward the ectoderm fate. This is evidenced by transient expression of FGF5 and uniform expression of Otx 2 and Sox 2 but lack of brachyury and $\alpha$-fetoprotein (AFP), mesoendodermal markers (4). Prolonged culture in the ESC growth medium will lead to the formation of "embryoid bodies", which is not ideal for neural differentiation.

Unlike most of the neural differentiation protocols that involve long-term aggregated culture or co-culture with stroma cells, in our protocol, the ESC aggregates are reseeded onto a culture dish (without feeder) and they reform individual monolayer colonies. This permits individual cells to be exposed to the culture environment evenly for synchronized differentiation. It also allows morphological observation. After 8-10 days of differentiation, neuroepithelia, characterized by columnar epithelial morphology, appear. Their identity is confirmed by expression of a host of neuroectodermal transcription factors including Pax6, Lhx2, Six3, and Sox2, and the lack of other germ layer markers (4). By two weeks of differentiation, these epithelial cells organize into rosettes, reminiscent of the cross section of the neural tube. These cells express Sox 1 in addition to the above neural markers and exhibit polarity by concentrated expression of $\mathrm{N}$-cadherin in the lumen $(3,4)$. This stage of differentiation can be subdivided into two morphologically distinct stages. The early columnar neuroepithelia, which we term primitive anterior neuroepithelia, uniformly express anterior transcription factors but can be readily specified to other regional identities (6-9). Therefore, the primitive neuroepithelia behave like true neural stem cells. We will use midbrain dopamine neuron differentiation to illustrate the point. The late columnar neuroepithelia, usually with a lumen inside and expression of definitive neural marker Sox 1, can not be patterned to region-specific progenitors and are termed as definitive neuroepithelia $(7,9)$.

The neuroepithelia in the form of neural tube-like rosettes can be readily detached from the culture surface and expanded in suspension like "neurospheres" although these cells are relatively primitive comparing to the progenitor aggregates derived from the embryonic or adult brain. These cells, upon dissociation and plating onto substrate, will differentiate to postmitotic neurons. 


\section{Materials}

\subsection{Supplies}

1. Polystyrene multidishes, 6-well and 24-well (Nunc; cat. no. 140675 and 142475).

2. $60 \times 15 \mathrm{~mm}$ Petri-dish (Fisher Scientific; cat. no. 08-757-13A)

3. T25 flasks, the polystyrene flasks with polyethylene filter cap (TPP, Trasadingen, Switzerland; cat. no. 90026).

4. T75 flasks, the polystyrene flasks with polyethylene filter cap (Nunc; cat. no. 156499).

5. Polystyrene conical tube, 15 - and 50-ml (Research Products International Corp; cat. no. 163224 and 163228.).

6. Serological pipettes 5-, 10- and 25-ml (Fisher Scientific; cat. no. 13-678-11D, 13-678-11E and 13-678-11).

7. 9" Pasteur pipettes (Fisher Scientific; cat. no. 13-678-20D).

8. Steriflip sterile disposable vacuum filtration system (Millipore, cat. no. SCGP00525).

9. Stericup vacuum driven disposable filtration system (Millipore, cat. no. SCGPU05RE).

\subsection{Stock solutions}

1. L-Glutamine solution (200 mM) (Gibco-BRL, Rockville, MD; cat. no. 25030). Make aliquots of $5 \mathrm{~mL}$ and store at $-20^{\circ} \mathrm{C}$.

2. MEM non-essential amino acids solution (Gibco-BRL; cat. no. 11140).

3. Knockout serum replacer (Gibco-BRL; cat. no. 10828). Store stock in $-80^{\circ} \mathrm{C}$. Make aliquots of $50 \mathrm{~mL}$ and store at $-20^{\circ} \mathrm{C}$ if it can not be used up in a week after thawed.

4. Dulbecco's modified eagle medium: Nutrient mixture F-12 1: 1 (DMEM/F12) (GibcoBRL; cat. no. 11330).

5. $\beta$-Mercaptoethanol (14.3 M) (Sigma; cat. no. M7522).

6. Neurobasal medium (Gibco-BRL; cat. no. 21103)

7. Recombinant human FGF basic (Invitrogen; cat. no. 13256-029) is dissolved in human ESC growth medium at a final concentration of $10 \mu \mathrm{g} / \mathrm{ml}$. Aliquot $100 \mu \mathrm{l}$ into sterilized tubes and store at $-80^{\circ} \mathrm{C}$. A final concentration of $4 \mathrm{ng} / \mathrm{ml}(1: 2500)$ is used to culture human ESCs.

8. Dispase solution (1.5 U/ml); dissolve $75 \mathrm{U}$ of Dispase (Gibco-BRL; cat. no. 17105-041) in $50 \mathrm{ml}$ DMEM/F12 in a water bath for $15 \mathrm{~min}$ and filter sterilize the dispase solution with a $50 \mathrm{ml}$ Steriflip.

9. Accutase in DPBS/0.5 mM EDTA (Innovative Cell Technology, San Diego, CA; cat. no. AT104).

10. $0.05 \%$ trypsin-EDTA solution (Gibco-BRL; cat. no 25300 ).

11. Trypsin inhibitor $(1 \mathrm{mg} / \mathrm{ml})$; dissolve $50 \mathrm{mg}$ trypsin inhibitor (Gibco-BRL; cat. no. 17075-029) in $50 \mathrm{ml}$ DMEM/F12 and filter through $50 \mathrm{ml}$-Steriflip.

12. Heparin ( $1 \mathrm{mg} / \mathrm{ml})$; dissolve $10 \mathrm{mg}$ heparin (Sigma; cat. no. H3149) in $10 \mathrm{ml}$ DMEM/ F12 medium. Aliquot $0.5 \mathrm{ml}$ into sterilized tubes and store at $-80^{\circ} \mathrm{C}$.

13. 100X N2 supplement (Gibco-BRL; cat. no. 17502-048). 
14. 50X B27 supplement without vitamin A (Gibco-BRL; cat. no. 12587-010)

15. Fetal bovine serum (FBS) (Gibco-BRL; cat. no. 16000-044).

16. Laminin from human placenta (Sigma; cat. no. L6274).

17. Ascorbic acid $(200 \mu \mathrm{g} / \mathrm{ml})$; dissolve $2 \mathrm{mg}$ ascorbic acid (Sigma; cat. no. A-4403) in $10 \mathrm{ml}$ PBS. Aliquot $0.5 \mathrm{ml}$ into sterilized tubes and store at $-80^{\circ} \mathrm{C}$.

18. Cyclic AMP (1mM); dissolve $4.914 \mathrm{mg}$ cyclic AMP (Sigma; cat. no. D-0260) in 10 $\mathrm{ml}$ sterilized water. Aliquot $0.5 \mathrm{ml}$ into sterilized tubes and store at $-80^{\circ} \mathrm{C}$.

19. Sonic hedgehog (SHH, $100 \mu \mathrm{g} / \mathrm{ml}$ ); dissolve $0.1 \mathrm{mg} \mathrm{SHH} \mathrm{(R \& D;} \mathrm{cat.} \mathrm{no.} \mathrm{1845-SH)}$ in $1 \mathrm{ml}$ sterilized PBS with $0.1 \%$ BSA. Aliquot $30 \mu \mathrm{l}$ into sterilized tubes and store at $-80^{\circ} \mathrm{C}$.

20. Recombinant BDNF, GDNF or IGF-1 (all are $100 \mu \mathrm{g} / \mathrm{ml}$ ); dissolve $200 \mu \mathrm{g}$ BDNF, GDNF or IGF-1 (Pepro Tech Inc; cat. no. 450-02, 450-10, 100-11) in $2 \mathrm{ml}$ sterilized water. Aliquot $30 \mu \mathrm{l}$ into sterilized tubes and store at $-80^{\circ} \mathrm{C}$.

21. Recombinant FGF8 (100 $\mu \mathrm{g} / \mathrm{ml})$; dissolve $200 \mu \mathrm{g}$ FGF8 (Pepro Tech Inc; cat. no. $100-25$ ) in $2 \mathrm{ml}$ sterilized PBS with $0.1 \%$ BSA and $2 \mu \mathrm{g} / \mathrm{ml}$ heparin. Aliquot $30 \mu \mathrm{l}$ into sterilized tubes and store at $-80^{\circ} \mathrm{C}$.

22. TGFß3 (10 $\mu \mathrm{g} / \mathrm{ml})$; dissolve $10 \mu \mathrm{g}$ TGF $\beta 3$ (R\&D; cat. no. 243-B3) in $1 \mathrm{ml}$ PBS. Aliquot $30 \mu \mathrm{l}$ into sterilized tubes and store at $-80^{\circ} \mathrm{C}$.

\subsection{Media}

1. Human ESC growth medium. Sterilely combine $392.5 \mathrm{ml}$ DMEM/F12, $100 \mathrm{ml}$ Knockout serum replacer, $5 \mathrm{ml}$ MEM non-essential amino acids solution, $2.5 \mathrm{ml}$ of $200 \mathrm{mM}$ L-glutamine solution (final concentration of $1 \mathrm{mM}$ ), and $3.5 \mu \mathrm{l} 14.3 \mathrm{M} \beta$ Mercaptoethanol (final concentration of $0.1 \mathrm{mM}$ ). Medium can be stored at $4^{\circ} \mathrm{C}$ for up to 7-10 d (see Note 1).

2. Neural induction medium (DMEM/F12/N2). Sterilely combine: $489 \mathrm{ml}$ of DMEM/ F12, $5 \mathrm{ml} \mathrm{N} 2$ supplement, $5 \mathrm{ml}$ MEM non-essential amino acids solution, and $1 \mathrm{ml}$ of $1 \mathrm{mg} / \mathrm{ml}$ Heparin. Medium can be stored at $4^{\circ} \mathrm{C}$ for up to $2 \mathrm{w}$.

3. Neural differentiation medium. Sterilely combine: $485 \mathrm{ml}$ of Neurobasal, $5 \mathrm{ml} \mathrm{N} 2$ supplement, and $10 \mathrm{ml} \mathrm{B} 27$ supplement. Medium can be stored at $4{ }^{\circ} \mathrm{C}$ for up to $4 \mathrm{w}$. Add laminin $(1 \mu \mathrm{g} / \mathrm{ml})$, cAMP (1:10000), ascorbic acid (1:1000), BDNF (1:10000), GDNF (1:10000) and IGF-1 (1:10000) before use.

\section{Methods}

\subsection{Make ESC Aggregates}

1. $10 \mathrm{~min}$ before start, warm up DMEM/F12, dispase and ES cell growth medium in $37^{\circ}$ C water bath.

2. Aspirate medium from each well of the 6-well plate containing the ESCs (see Fig. 1A and Note 2).

3. Wash the cells with DMEM/F12 once to remove serum replacement.

\footnotetext{
${ }_{1}$ If filtration is needed, we recommend using disposable receipt bottle (Stericup, Millipore) other than reused glass bottle.

2 The undifferentiated state of ESCs is very important for efficient neural induction. If there are differentiated cells in the ESC culture, floating ESC aggregates are easy to attach to the flask and neurons will be easily found in the culture dish when these aggregates are plated down (see Fig1 H and I)
} 
4. Aspirate DMEM/F12 and add to each well $1 \mathrm{ml}$ dispase.

5. Incubate in a $\mathrm{CO}_{2}$ incubator for $3 \mathrm{~min}$; observe the cells under a phase contrast microscope.

6. When the edges of ESC colonies begin to curl, aspirate the dispase-containing medium from the 6-well plate and wash the cells gently with $2 \mathrm{ml}$ DMEM/F12.

7. Collect the ESC colonies using a 10-ml serological pipette. If some ESC colonies remain attached, blow the colonies off gently and pool all the cells to a 50-ml conical tube.

8. Pipette up and down against the bottom of the tube to break up the colonies into 200 $\mu \mathrm{m}$ pieces (see Note 3 ).

9. Centrifuge at $200 \mathrm{~g}$ for $1 \mathrm{~min}$ at room temperature.

10. Aspirate the medium off the cell pellet.

11. Resuspend the ESCs in the ESC growth medium and transfer them to flasks (for one entire 6-well plate resuspend the cells in $50 \mathrm{ml}$ and culture them in a T75 flask; for 1-3 wells of cells use $15 \mathrm{ml}$ ESC growth medium and transfer to a T25 flask).

12. Within 12-18 hours, replace most of the medium to remove dead cells, and switch cells to a new flask to remove attached MEF if necessary.

13. Continue feeding the ESC aggregates every day for a total of 4 days. Set the flask down at a tilted angle so that the ESC aggregates settle in one corner of the flask. Aspirate off about half of the medium and add fresh ESC growth medium to replace the amount aspirated.

\subsection{Neuroepithelial Differentiation (Formation of Neural Tube-Like Rosettes)}

1. After 4 days, aspirate the ESC growth medium completely after the ESC aggregates are settled down to the corner of the flask. Rinse the aggregates with the neural induction medium once and resuspend the aggregates in the neural induction medium.

2. Change half of the medium 2 days later.

3. The next day, pre-coat the 6-well plate with $300 \mu \mathrm{l}$ neural induction medium supplemented with $20 \mu \mathrm{g} / \mathrm{ml}$ laminin each well (see note 4). For polyornithine-treated coverslips in 24-well plates, coat with $50 \mu \mathrm{l}$ neural induction medium/laminin (see Note 5).

4. Transfer the ESC aggregates into a $50 \mathrm{ml}$ conical tube and let the aggregates settle to the bottom of the tube. Aspirate off most of the medium and transfer all the cell aggregates to a 60-mm Petri dish. Get rid of abnormal aggregates, such as pigmented and strange-shaped ones (see Fig. 1H).

5. Plate 30-40 aggregates to each well of precoated 6-well plate or 3-4 aggregates on each coverslip in a 24-well plate and let the aggregates attach overnight.

\footnotetext{
${ }^{3}$ Usually the size of the clusters is around twice the size for ESCs for passaging/splitting. Cells will die and the yield of ESC aggregates will be quite low if the size of the clusters is too small. The differentiation will be unsynchronized if the size is too large.

${ }^{4} \mathrm{As}$ an alternative to the attachment of ESC aggregates using laminin, FBS can also be used. Add neural induction medium containing $10 \%$ FBS to the ESC aggregates as mentioned in subheading 3.2. Plate 1-1.5 ml of medium containing 30-40 aggregates to each well of a 6-well plate and let the cells attach in the incubator overnight. Right after the cells attach, wash the cells with warm DMEM/F12 once and feed with neural induction medium. Please note that it is better to leave the cells in the presence of serum for as short time as possible. ${ }^{5}$ Coverslips need be pretreated with nitric acid for 1 hour and then $95 \%$ ethanol for $30 \mathrm{~min}$ after washing with distilled water. These coverslips then need be coated with $0.1 \mathrm{mg} / \mathrm{ml}$ polyornithine overnight before use (for details see www.wicell.org).
} 
6. Aspirate the medium containing laminin and add $2 \mathrm{ml}$ neural induction medium to each well of the 6-well plate or $0.5 \mathrm{ml}$ to each well of a 24-well plate on the next day.

7. Change most medium every other day.

8. Observe the culture daily. Cells in the colony will become elongated in 2-3 days after attachment and will arrange radically like rosettes in the center with a few flat cells in the periphery (see Fig. 1C). At this period, we call them primitive anterior neuroepithelia. With further culturing in the same medium for another $4-5 \mathrm{~d}$, the area and thickness of rosettes increase and almost every colony contains multiple neural tube-like rosettes with lumens, we call them definitive neuroepithelia (see Fig. 1D).

\subsection{Enrichment and Expansion of Neuroepithelial Cells}

1. After formation of neural tube-like rosettes at day 14-15, the multi-cell layered structures need to be subcultured in order to avoid cell death and to promote cell growth.

2. Aspirate off old medium and add $1 \mathrm{ml}$ new neural induction medium to each well.

3. Gently blow off the clusters with a 5-ml serological pipette and collect them to a 15ml conical tube.

4. Triturate the clumps with a 5- or 10-ml serological pipette up and down twice, but not to break up the clumps.

5. Transfer the floating cells (mostly aggregates of neural rosette cells) to a new tube. Collect the large clumps that are settled on the bottom of the tube to a 1.5 eppendorf tube and use a P200 pipette to break them into small clusters.

6. Pool all the cells together and centrifuge at $50 \mathrm{~g}$ for $2 \mathrm{~min}$ at room temperature.

7. Resuspend the cell pellets with $15 \mathrm{ml}$ neural induction medium supplemented with B27 (1X) and transfer them to a T25 flask.

8. The rosette aggregates will roll up to form round spheres after about $1 \mathrm{~d}$. (see Fig. $1 \mathrm{E})$.

9. Change half of the medium and feed the neuroepithelial aggregates every other day with the neural induction medium with B27.

\subsection{Differentiation of Neurons from neural progenitors}

1. The neuroepithelial aggregates can be kept in suspension for several weeks or months (see note 6). How long the neuroepithelial cells should be expanded in suspension depends on research objectives. They usually generate large projection neurons in the first month, followed by smaller interneurons, astocytes, and oligodendrocytes.

2. Pre-coat culture plates or polyornithine-treated coverslips with laminin $(20 \mu \mathrm{g} / \mathrm{ml}$, diluted in neural differentiation medium) at $37^{\circ} \mathrm{C}$ for at least 3 hours. For coverslips in 24-well plates, coat one coverslip with $50 \mu 1$ laminin medium. For 1 well of 6-well plates, coat with $300 \mu \mathrm{l}$ laminin medium in the center.

3. Warm up accutase, trypsin, trypsin inhibitor, DMEM/F12 and neural differentiation medium in $37^{\circ} \mathrm{C}$ water bath.

\footnotetext{
${ }^{6}$ Flame polish a cotton plugged Pasteur pipette to narrow the opening end slightly and make a $20-30^{\circ}$ bend at the narrow part of the shaft. Pipette up and down 1-3 times to let the aggregates go through the bended pipette and shear them into small pieces. In this way, neuroepithelial aggregates can be expanded from 1 flask into 2 .
} 
4. Collect the neuroepithelial aggregates into a $15-\mathrm{ml}$ conical tube and let the aggregates settle to the bottom of the tube. Aspirate off most of the medium and transfer all the aggregates to a $1.5 \mathrm{ml}$ eppendorf tube. Gently remove the remaining medium.

5. Add $500 \mu \mathrm{l}$ accutase/trypsin (1:1) to the cells and incubate at $37^{\circ} \mathrm{C}$ for $2-3 \mathrm{~min}$.

6. Add $500 \mu \mathrm{l}$ trypsin inhibitor to the cells and centrifuge at $50 \mathrm{~g}$ for $2 \mathrm{~min}$ at room temperature.

7. Remove supernatant and wash with DMEM/F12 once.

8. Add $100-200 \mu 1$ neural differentiation medium to the tube and pipette up and down against the bottom of the tube to break up the cells into small clusters $(50-100 \mu \mathrm{m})$ or single cells with a P200 pipette (see note 7).

9. Plate the cells in the pre-coated plates or coverslips. Check under the microscope about the density of the cells. Similar to seeding ESC aggregates, plate 30-40 aggregates in each well of a 6-well plate or 3-4 aggregates on each coverslip. For single cell plating, resuspend the cells in neural differentiation medium at the density of 400,000 cells $/ \mathrm{ml}$. Then put $25 \mu \mathrm{l}$ cells onto each coated coverslip or $150 \mu \mathrm{l}$ onto each well of 6-well plate.

10. After the cells attach to the bottom (2-3 hours later after plating), add $1 \mathrm{ml}$ or $3 \mathrm{ml}$ neural differentiation medium to each well of the 24-well plates or 6-well plates, respectively.

11. Feed the cells every other day with the same medium and change half of the medium each time.

\subsection{Differentiate Human ESCs to Midbrain Dopaminergic Neurons}

1. Differentiate primitive neuroepithelial cells from hESCs as described in 3.1-3.3. This usually happens at around 8-10d after differentiation from hESCs, with the appearance of columnar cells in the culture.

2. Aspirate off old medium and add $3 \mathrm{ml}$ neural induction medium supplemented with FGF8b (50 ng/ml) and SHH (100 ng/ml).

3. Continue feeding the cells every other day with the same medium with FGF8b and SHH for 7 days until formation of neural tube-like rosettes.

4. Enrich and expand the neuroepithelia as described above (3.3) but in neural induction medium containing FGF8b (50 ng/ml), SHH (100 ng/ml), B27 (1X), and ascorbic acid $(200 \mu \mathrm{M})$.

5. Feed the neural progenitor aggregates every other day with the neural induction medium with FGF8b, SHH, B27 and ascorbic acid.

6. After 7 days of culture in suspension, the neural progenitor aggregates are dissociated to single cells after digestion with accutase/trypsin (1:1) and plated onto laminin precoated surface as mentioned above (3.4).

7. Feed the cells every other day with the neural differentiation medium containing FGF8b (50 ng/ml), SHH (100 ng/ml), ascorbic acid $(200 \mu \mathrm{M})$, cAMP $(1.0 \mu \mathrm{M})$, TGFß3 (1 ng/ml), BDNF (10 ng/ml), GDNF (10 ng/ml) and Wnt3a conditioned medium (1X) for 3 weeks (see note 8).

${ }^{7}$ Cells can be dissociated to either single cells or small clusters. Small clusters tend to survive better than single cells. For dopaminergic differentiation, we dissociate the cells to single cells and plate at a high density. 
8. Withdraw FGF8b, SHH and Wnt3a conditioned medium at day 44 after differentiation and maintain the cells in neural differentiation medium with ascorbic acid, cAMP, TGFß3, BDNF and GDNF.

9. At this point, numerous multipolar dopaminergic neurons can be detected through immunostaining with TH antibody.

\section{Acknowledgments}

This study was supported by the National Institute of Neurological Disorders and Stroke (NS045926, NS046587), the Michael J. Fox Foundation, and partly by a core grant to the Waisman Center from the National Institute of Child Health and Human Development (P30 HD03352).

\section{References}

1. Zhang SC. Embryonic stem cells for neural replacement therapy: Prospects and Challenges. J. Hematother. Stem Cell Res 2003;12:625-634. [PubMed: 14977472]

2. Du ZW, Zhang SC. Neural differentiation from embryonic stem cells: which way? Stem Cell Development 2004;13:372-381.

3. Zhang SC, Wernig M, Duncan ID, Brüstle O, Thomson JA. In vitro differentiation of transplantable neural precursors from human embryonic stem cells. Nat. Biotechnol 2001;19:1129-1133. [PubMed: 11731781]

4. Pankratz MT, Li XJ, Lavaute TM, Lyons EA, Chen X, Zhang SC. Directed neural differentiation of human embryonic stem cells via an obligated primitive anterior stage. Stem Cells 2007;25:1511-1520. [PubMed: 17332508]

5. Hemmati-Brivanlou A, Melton D. Vertebrate embryonic cells will become nerve cells unless told otherwise. Cell 1997;88:13-17. [PubMed: 9019398]

6. Li XJ, Du ZW, Zarnowska ED, Pankratz M, Hansen LO, Pearce RA, Zhang SC. Specification of motoneurons from human embryonic stem cells. Nat Biotechnol 2005;23:215-221. [PubMed: 15685164]

7. Li XJ, Hu BY, Jones SA, Zhang YS, Lavaute T, Du ZW, Zhang SC. Directed differentiation of ventral spinal progenitors and motor neurons from human embryonic stem cells by small molecules. Stem Cells 2008;26:886-893. [PubMed: 18238853]

8. Yang D, Zhang ZJ, Oldenburg M, Ayala M, Zhang SC. Human embryonic stem cell-derived dopaminergic neurons reverse functional deficit in parkinsonian rats. Stem Cells 2008;26:55-63. [PubMed: 17951220]

9. Yan Y, Yang D, Zarnowska ED, Du Z, Werbel B, Valliere C, Pearce RA, Thomson JA, Zhang SC. Directed differentiation of dopaminergic neuronal subtypes from human embryonic stem cells. Stem Cells 2005;23:781-790. [PubMed: 15917474]

${ }^{8} \mathrm{~L}$ cells that express Wnt3a (ATCC, Manassas, VA) are conditioned in neural differentiation medium for 24 hours and the conditioned medium is used at a final concentration of $50 \%$ from day 24 to day 44 to promote the proliferation of specified DA progenitors. 


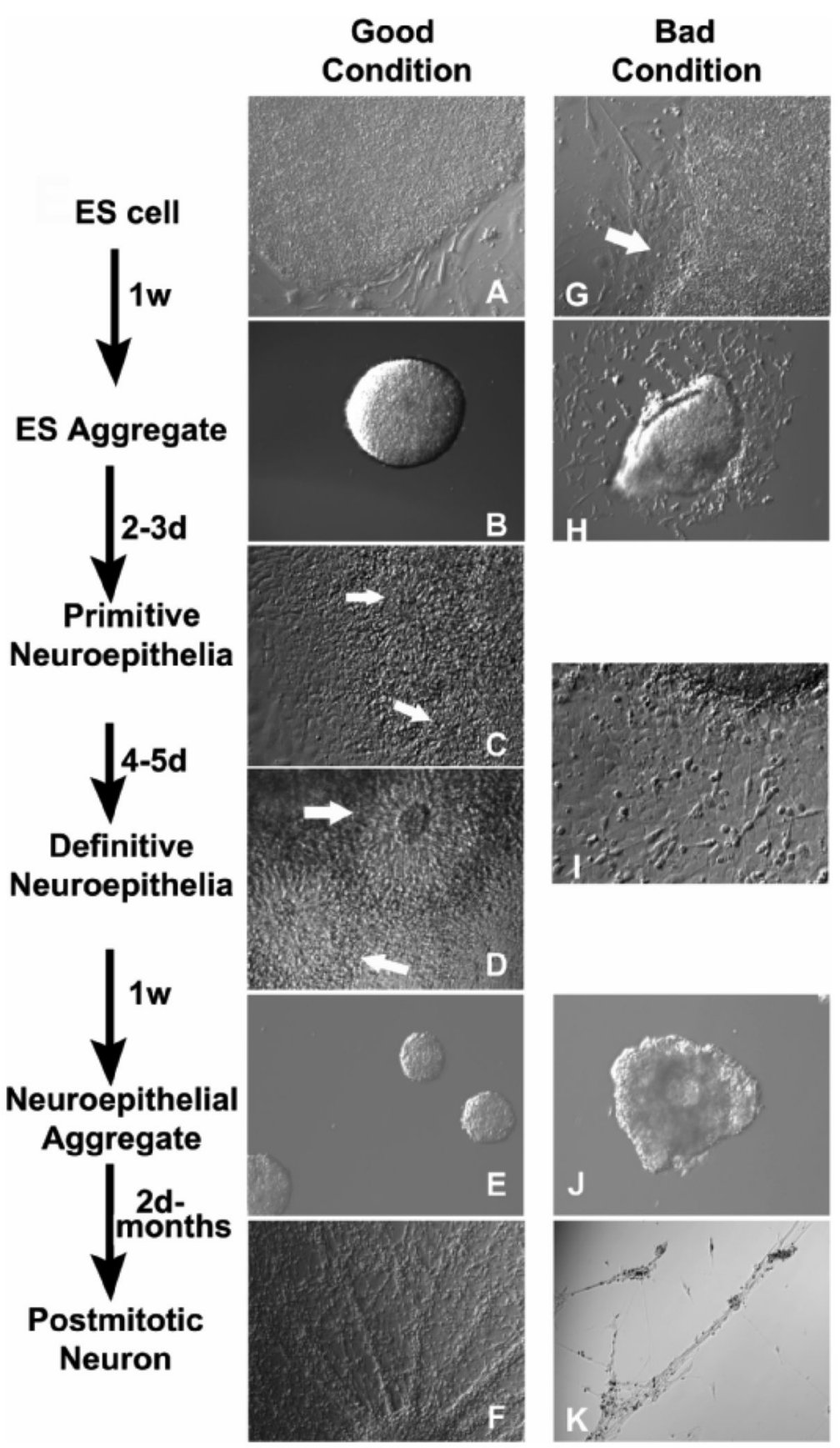

Fig. 1.

Five morphologically distinct steps of neuronal differentiation from human ESCs. (A-F) Phase contrast image showing ESC colony, ESC aggregate, primitive neuroepithelia, definitive neuroepithelia, neuroepithelial aggregate and postmitotic neuron stages. Arrows show rosette formation in primitive or definitive neuroepithelial stages. (G-K) Phase contrast image showing a partially differentiated ESC colony (arrow), differentiated ESC aggregates, asynchronized neural differentiation with neurons appearing at the neuroepithelial stage, neuroepithelial aggregates and postmitotic neurons. 